\title{
Weed Management in Vegetable Crops to Reduce the Yield Losses
}

\author{
Mandeep Singh ${ }^{1 *}$, Amit Kaul ${ }^{2}$, Vivek Pandey ${ }^{3}$ and Avtar Singh Bimbraw ${ }^{4}$ \\ ${ }^{1}$ Krishi Vigyan Kendra, Gurdaspur, Punjab Agricultural University, Ludhiana, India \\ ${ }^{2}$ Krishi Vigyan Kendra, Pathankot Punjab Agricultural University, Ludhiana, India \\ ${ }^{3}$ Regional Research Station, Gurdaspur, Punjab Agricultural University, Ludhiana, India \\ ${ }^{4}$ Department of Agronomy, Punjab Agricultural University, Ludhiana, India \\ *Corresponding author
}

\section{A B S T R A C T}

Weed is a plant growing where it is not desired so weeds are ubiquitous. The vegetable fields are usually infested by a wide spectrum of broad and grassy weeds. Weeds compete with the

\section{Keywords}

Herbicides,

Vegetables,

Weedicides, Weed

control method,

Weeds, Yield losses

\section{Article Info}

Accepted:

10 June 2019

Available Online:

10 July 2019

crops for water, soil, nutrients, light, space and crop yields are significantly reduced as reported by several studies. The vegetable as well as agricultural crops are infested with a variety of weeds. Out of over 800 weeds documented in India, 80 are considered as serious and nearly 200 as major weeds. They also harbour many insect-pests and microorganisms. On an average weed extract two times more $\mathrm{N}$ and $\mathrm{Ca}$ and 25 per cent more potassium than the crop as observed in some studies. Reduction in economic yield of vegetables has been reported to be 682 per cent in potato, $25-30$ per cent in peas, $70-80$ per cent in carrot, 67 per cent in onion, 4271 per cent in tomato and 61 per cent in cauliflower. Farmers usually have a various methods for weed control and their choice of control measures is closely controlled by economics. Although, herbicides were introduced to horticultural crops in India in 1960, hand tools and animal drawn equipment still remain important methods of weed control in vegetable crops. Conventional methods of weed control have become an expensive input in the cultivation of vegetable crops. Owing to high cost and non- availability of labour at proper time therefore, no single method of weed control is adequate and cost effective. However, integrated weed management is a systematic approach to minimize weed impacts and optimize the land use by the different weed management practices.

\section{Introduction}

Weeds are ubiquitous. The vegetable fields are usually infested by a wide spectrum of broad and grassy weeds. Weeds compete with the crops for water, soil; nutrients, light and space thus reduce crop yields up to 37 per cent (Varshney, 2007). The vegetable as well as agricultural crops are infested with a variety of weeds. Out of over 800 weeds documented in India, 80 are considered as serious and nearly
200 as major weeds. They also harbour many insect-pests and microorganisms (Cooper and Harrison, 1973). On an average weed extract two times more $\mathrm{N}$ and $\mathrm{Ca}$ and 25 per cent more potassium than the crop (Mallik et al., 1998). Reduction in economic yield of vegetables has been reported to be 6-82 per cent in potato, 25-30 per cent in peas, 70-80 per cent in carrot, 67 per cent in onion, 42-71 per cent in tomato and 61 per cent in cauliflower (Singh, 1980). Farmers usually 
have a variety of methods for weed control and their choice of control measures is closely controlled by economics. Although, herbicides were introduced to horticultural crops in India in 1960, hand tools and animal drawn equipment still remain important methods of weed control in vegetable crops.

Conventional methods of weed control have become an expensive input in the cultivation of vegetable crops. Owing to high cost and non- availability of labour in time and no single method of weed control is adequate or cost effective. Integrated weed management is a systematic approach to minimize weed impacts and optimize the land use by the different weed management practices (Aldrich, 1984) (Table 1-14).

\section{Definition of weed}

Many Scientist defined weed few of them are as follows:-

A plant out of place or growing where it is not desired (Blatchley, 1912)

The plants which are a nuisance (Harper, 1960)

A plant growing where we do not want it (Salisbury, 1961)

A plant out of place (Kalingman, 1961)

\section{Characteristics of weeds}

Weeds have rapid seedling growth and ability to reproduce when young. Redroot pigweed can flower and reproduce seed when less than 8 inches tall.

Have quick maturation or only a short time in vegetative phase.

Have dual mode of reproduction
Often self compatible but self pollination is not obligatory

Weeds have environmental plasticity. Many weed are capable of tolerating and growing under a wide range of climatic and edaphic conditions.

Resist detrimental environmental factors. Weed seeds resist decay for long periods in soil and remain dormant.

Often produce seed the same size and shape as crop seed, making physical separation difficult and facilitating spread by man.

Some annual weeds produce more than one seed crop per year and seed is produced as long as growing conditions permits.

Many weeds have specially adapted long and short range seed dispersal mechanisms. Roots of some weeds are able to penetrate and emerge from deep in soil

Many weeds have adaptations that repel grazing, such as spines, taste or odour.

Weeds have great competitive ability for nutrients, light and water and can compete by special means (e.g. rosette formation, climbing growth, allelopathy)

Weeds resist control, including herbicides

\section{Harmful aspects of weeds}

Plant competition. Weeds compete with crop plant for nutrients, water and light.

Weeds increase protection costs because they harbor other pests.

Increased production and processing costs. Any weed control operation from hand hoeing to herbicide application, cost money. 
Decreased land value and reduced crop choice.

Reduced quality of farm products. Most seed in vegetable crops perpetuate the problem when the crop seed is replanted.

Some weeds causes cattle death because cattle like it and often eat it selectively e.g. larkspur, locoweeds, crazyweeds etc. It may cause some allergic problem to human also such as runny nose, sneezing etc.

Approaches for integrated weed
management

Preventive

Cultural

Chemical

Biological

\section{Preventive weed management}

Use clean seed. As many weed seeds get mixed with the main crop and these seeds should be separate before sowing to avoid weeds.

Clean tillage implements. Many weed seeds stick to the implements used in the previous crops, so before using these implements these should be thoroughly cleaned.

Avoid use/transportation of soil from weed infested area

Prevent reproduction of weeds by removing then in vegetative stage.

Use weed seed screen filter irrigation water to avoid weed seeds dispersal through irrigation water.

Restrict live stock movement to non weed infested area. Many weed seeds get stick to live stock and
Use thoroughly decomposed organic manure, because many seeds remains vival in the cattle dung and if it is used in the fields undecomposed then these weed seeds will germinate.

Weeds should be removed from the fields before the critical crop weed competition period to avoid yield losses.

\section{Cultural practices}

Stale seed bed

Mixed cropping

Land preparation

Mulching

Hand weeding

Burning and flaming

Crop Rotation

Irrigation

Solarization

\section{Stale seed beds}

Stale ('false') seed beds are sometimes used for vegetables when other selective weedcontrol practices are limited or unavailable.

Success depends on controlling the first flush of emerged weeds before crop emergence, and on minimal disturbance, which reduces subsequent weed flushes. Basically, this technique consists of the following:

Preparation of a seedbed 2-3 weeks before planting to achieve maximum weed-seed germination near the soil surface.

Planting the crop with minimum soil disturbance to avoid exposing new weed seed to favourable germination conditions.

Treating the field with a non-residual herbicide to kill all germinated weeds (William et al., 2000) just before or after planting, but before crop emergence. 


\section{Crop rotation}

Crop rotation is the programmed succession of crops during a period of time in the same plot or field. It is a key control method to reduce weed infestation in vegetables. Crop rotation was considered for a long time to be a basic practice for obtaining healthy crops and good yields. This concept was mistakenly eliminated with the use of more agrochemicals. At present, however, crop rotation is gaining interest and is of value in the context of integrated crop management. Classically, crop rotations are applied as follows:

Alternating crops with a different type of vegetation: leaf crops (lettuce, spinach, cole), root crops (carrots, potatoes, radish), bulb crops (leeks, onion, garlic), fruit crops (squash, pepper, melon).

Alternating grass and dicots, such as maize and vegetables.

Alternating different crop cycles: winter cereals and summer vegetables.

Avoiding succeeding crops of the same family: Apiaceae (celery, carrots), Solanaceae (potato, tomato).

Alternating poor- (carrot, onion) and highweed competitors (maize, potato).

Avoiding problematic weeds in specific crops (e.g. Malvaceae in celery or carrots, parasitic and perennials in general).

Examples of crop rotations are as follow (Zaragoza et al., 1994):

\section{In temperate regions}

Pepper - onion - winter cereal

Melon - beans - spinach - tomato
Tomato - cereal - fallow

Lettuce - tomato - cauliflower

Potato - beans - cole - tomato- carrots

Melon - artichoke - beans - red beet - wheat cole

\section{In tropical regions}

Tomato - okra - green bean

Sweet potato - maize - mung bean

Introducing a fallow in the rotation is essential for the control difficult weeds (e.g. perennials), cleaning the field with appropriate tillage or using a broad-spectrum herbicide. It is also important to avoid the emission of weed seeds or other propagules.

\section{Mixed cropping}

Growing two or more crops at the same time and adjacent to one another is called mixed cropping, or intercropping. Crop cycles must coincide totally or partially (relay-cropping).

The advantages are a better use of space, light and other resources, a physical protection, a favourable thermal balance, better plant defense against some pests and fewer weed problems because the soil is better covered.

Inconveniences are intercrop competition, difficult management and mechanization, a greater need for hand-labour, incomplete control of weeds. Sometimes the results are less productive than cultivating just one crop alone.

Usually the 'companion' crops are fast and low-growing plants, creeping and erect plants, or symbiotic species. Some examples are:

In temperate regions:

Lettuce + carrots;

Cole crops + leeks, onion, celery, tomato;

Maize + beans, soybean 
In tropical regions: this technique is very well adapted to the traditional agricultural system:

Maize + beans + squash, manioc;

Tomato + pigeon pea, manioc;

Sugar cane + onion, tomato.

\section{Soil solarization}

Soil solarization is a preventive method that exploits solar heating to kill weed seeds and therefore reduce weed emergence. High soil temperature, if lasting long enough, is able to kill the reproduction structures of pests, diseases, and weeds. Solarization can be defined as a soil disinfection method that exploits the solar energy available during the warmest period of the year. To increase the solarization effect as much as possible, the soil surface must be smooth and must contain enough water to favour heat transfer down the profile and to make reproductive structure of pests, diseases and weeds more sensitive to heat damage. For this reason, prior to solarization the soil is usually irrigated and a plastic mulch film is laid down onto the soil to further increase soil heating and to avoid heat dissipation to the atmosphere. The success of soil solarization as a weed control method does not depend on the actual value of peak temperature reached in the soil but rather on temperature duration above a certain threshold $\left(45^{\circ} \mathrm{C}\right)$ on a daily basis (Horowitz et al., 1983). It follows that soil solarization can only be used in warm climates or under glasshouse conditions in warm-temperate and Mediterranean climates. For example, a significant reduction in weed emergence was observed over the following 12 months after one-month's solarization in a tunnel glasshouse used for vegetable production in Central Italy (Temperini et al., 1998). To retain as much as possible the weed control effect of solarization, the soil must not be cultivated subsequently because otherwise weed seeds present in deeper soil layers (less affected by heating) are brought up to the soil surface and can germinate.

\section{Land preparation and tillage}

As Labrada (1996) stated, suitable land preparation depends on a good knowledge of the weed species prevalent in the field. When annual weeds are predominant (Crucifers, Solanum, grass weeds) the objectives are unearthing and fragmentation. This must be achieved through shallow cultivation. If weeds have no dormant seeds (Bromus spp.), deep ploughing to bury the seeds will be advisable. If the seeds produced are dormant, this is not a good practice, because they will be viable again when they return to the soil surface after further cultivation. When perennial weeds are present, adequate tools will depend on the types of rooting. Pivot roots (Rumex spp.) or bourgeon roots (Cirsium spp.) require fragmentation and this can be achieved by using a rotovator or cultivator. Fragile rhizomes (Sorghum halepense) require dragging and exposure at the soil surface for their depletion, but flexible rhizomes (Cynodon dactylon) require dragging and removal from the field. This can be done with a cultivator or harrow. Tubers (Cyperus rotundus) or bulbs (Oxalis spp.) require cutting when rhizomes are present and need to be dug up for exposure to adverse conditions (frost or drought). This can done with mouldboard or disk ploughing. Chisel ploughing is useful for draining wet fields and reducing the infestation of deep-rooted hygrophilous perennials (Phragmites, Equisetum, Juncus). This is why reliable weed information is always necessary.

The success of many weed-control operations depends upon the timing of its implementation. The opportunity for mechanical operation is indeed essential. Action must be taken against annual weeds before seed dispersion takes place. 
Good practices in mechanical operations must look at optimal conditions, including the following:

Planting density must be in function of the weeding-tool working width.

Choice of adequate tools necessary for the work.

Paying attention to the weed and crop stage and avoiding delays in interventions.

Regulating the work depth, advance speed, attack angle.

Moisture content is important; look for the right tilth.

Do not increase the soil erosion: avoid parallel tillage to the slope direction line. Foresee climatic conditions after completion of work. Avoid tillage if rainfall is expected.

Another typical operation that requires mechanical tillage is herbicide soil incorporation. Some very volatile herbicides commonly used in vegetables (e.g. trifluraline) must be thoroughly incorporated in the soil at an adequate depth $(5-7 \mathrm{~cm})$. The implement used for herbicide incorporation must be in good condition. For example, rotavator blades must be sharpened. L-shaped blades are the best choice for chemical incorporation. For correct incorporation the soil must be neither too wet nor too dry. In the first case it is convenient to change the rotavator by a flexible or rigid tine harrow. Unbroken pieces of manure or soil clods can reduce the treatment efficacy (Kempen, 1989).

\section{Hand weeding}

It is practical and efficient method of weed control. It is very efficient for annual weeds, but not for perennial capable of vegetative reproduction, because root separate from shoot that then produce a new shoot. A disadvantage is that hand pulling does not the job done when it is most needed. Hand hoeing control the persistent perennials if it is done often enough. Although efficient and widely used, it takes a lot of time and human energy.

\section{Flaming}

Many plant processes are susceptible to high temperature disruption is attributed to coagulation and denaturation of protein, increasing membrane permeability and enzyme inactivation. Photosynthesis is decreased or stopped. Thermal death points for most plant tissue are between $45-55{ }^{\circ} \mathrm{c}$ after prolonged exposure. A flamer directs a petroleum based fuel emitted under pressure and ignited. Plant size at treatment influences efficacy much more than plant density. Required dose increased with plant growth stage and some species of annual weeds are more tolerant than others. The most tolerant species cannot be controlled with one flaming, regardless of dose.

\section{Mulching}

It excluded light and prevents shoot growth. Thick, wide mulches are required to control perennials that creep to the edge of a mulch and emerge. Mulches increase soil temperature and many promote better plant growth. Several different materials have been used to mulch, including straw, hay, manure, paper and black plastic. Mulches are used in high value crops.

\section{Irrigation}

Different methods of irrigation are used in vegetables. Drip irrigation is most efficient results in minimum weed population and maximum yield than the other methods (furrow, flood etc.). 
Table.1 Plant disease harbored by specific weeds

\begin{tabular}{|l|l|l|l|}
\hline Plant disease & Weed host & Crop Infested & Reference \\
\hline Black leg & $\begin{array}{l}\text { Black nighthade, } \\
\text { Common } \\
\text { lambsqurters, Redroot } \\
\text { pigweed }\end{array}$ & Potato & $\begin{array}{l}\text { Dallyn and } \\
\text { Sweet, 1970 }\end{array}$ \\
\hline Wilt disease & $\begin{array}{l}\text { lambsquarters, } \\
\text { Purselane, common } \\
\text { Redroot pigweed }\end{array}$ & Potato & Oshima et al., 1963 \\
\hline $\begin{array}{l}\text { White rust, early } \\
\text { Blight, Leaf spots, } \\
\text { Vascular wilts, } \\
\text { Cottony rot, White } \\
\text { mold }\end{array}$ & $\begin{array}{l}\text { Redroot pigweed } \\
\text { Stem rust, Leaf spot, } \\
\text { Leaf blight }\end{array}$ & $\begin{array}{l}\text { Potato, Tomato, } \\
\text { Beans, Cabbage }\end{array}$ & Commerce, 1967 \\
\hline
\end{tabular}

Table.2 Insects harbored by specific weeds

\begin{tabular}{|l|l|l|l|l|}
\hline \multicolumn{1}{|c|}{ Insect } & \multicolumn{1}{|c|}{ Vector } & \multicolumn{1}{c|}{ Weed host } & \multicolumn{1}{c|}{$\begin{array}{c}\text { Crop } \\
\text { infested }\end{array}$} & \multicolumn{1}{c|}{ Reference } \\
\hline $\begin{array}{l}\text { Colorado potato } \\
\text { beetle }\end{array}$ & Blackleg & $\begin{array}{l}\text { Common lamb } \\
\text { squarter }\end{array}$ & Potato & Bonde, 1939 \\
\hline $\begin{array}{l}\text { Beet leaf } \\
\text { hopper, Corn } \\
\text { borer }\end{array}$ & Curly top & Russian thistle & Sugarbeet & Brues, 1947 \\
\hline
\end{tabular}

Table.3 Weed flora associated with vegetable crops

In Rabi season

\begin{tabular}{|l|l|l|}
\hline Botanical name & Common name & Family \\
\hline Chenopodium album & Bathu & Chenopodiaceae \\
\hline Melilotus indica & Senji Methi & Papilionaceae \\
\hline M. Alba & Ban methi & Papilionaceae \\
\hline Lathyrus aphaca & Maturi, Pipura Pipari & Papilionaceae \\
\hline Vicia sativa & Anhta ankari & Papilionaceae \\
\hline Convolvulus arvensis & Hirankhuri & Convolvulaceae \\
\hline Rumex maritimus & Panbheri & Polygonaceae \\
\hline Cynodon dactylon & Bermuda grass, Doob grass & Graminae \\
\hline Cyperus rotundus & Motha & Cyperaceae \\
\hline Orobanche spp. & ----- & Orobanchaceae \\
\hline Spergula arvensis & Bhandhania & Caryophyllaceae \\
\hline Euphorbia hirta & Bari Dudhi & Euphorbiaceae \\
\hline
\end{tabular}


In Kharif season

\begin{tabular}{|l|l|l|}
\hline Botanical name & Common name & Family \\
\hline Trianthema portulacastrum & Patharchata, Gadhupura & Azoiaeae \\
\hline Echinochloa colona & Barnyard Grass, Sama grass, & Graminae \\
\hline Cyperus rotundus & Motha & Cyperaceae \\
\hline Digeria arvensis & ---- & Amaranthaceae \\
\hline Amaranthus viridis & Jangli Chaulai & Amaranthaceae \\
\hline Physalis minima & Ban Makaya & Solanaceae \\
\hline Phyllanthus niruri & $\begin{array}{l}\text { Haizardana Jar-Amla bhuin } \\
\text { Anmala }\end{array}$ & Euphorbiaceae \\
\hline Commelina benghalensis & Kanna, Kena & Commelinaceae \\
\hline Eleusine indica & Malanpuri Kodai & Graminae \\
\hline Ageratum conyzoides & Neela phool & Compositae \\
\hline Cynodon dactylon & Bermuda grass, Doob grass & Graminae \\
\hline Celosia argentea & $\begin{array}{l}\text { Safed murge ka phool, } \\
\text { Suawari }\end{array}$ & Amaranthaceae \\
\hline & & \\
\hline
\end{tabular}

Table.4 Crop losses due to weeds: It has estimated that crop losses caused by weeds are maximum in all pests (Varshney, 2009)

\begin{tabular}{|l|c|}
\hline \multicolumn{1}{|c|}{ Pest } & Share of losses (\%) \\
\hline Weeds & 37 \\
\hline Insects & 22 \\
\hline Diseases & 29 \\
\hline Rodents and others & 12 \\
\hline
\end{tabular}

Table.5 Viable weed seeds in manure sample

\begin{tabular}{|l|c|}
\hline Weed species & No. of Seeds/tones \\
\hline Amaranthus spinosus $\boldsymbol{L}$. & 2700 \\
\hline Echinochloa cruss-galli $\boldsymbol{L}$. & 1900 \\
\hline Stellaria media L. & 3500 \\
\hline Setaria viridis L. & 1600 \\
\hline S. glauca L. & 4500 \\
\hline Polygonum aviculare & 2700 \\
\hline Chenopodium album & 42000 \\
\hline Dactylis glomerata & 4700 \\
\hline Amaranthus retroflexus & 5200 \\
\hline Plantago major & 5100 \\
\hline Circium arvensis & 2700 \\
\hline Panicum capillare & 2100 \\
\hline Brassica kaber & 4700 \\
\hline
\end{tabular}

Source: Pleasant and Schiather (1994) 
Table.6 Critical stages for crop-weed competition in vegetable crops

\begin{tabular}{|l|c|l|}
\hline Crops & Critical stage (DAS/DAP) & Developmental stage \\
\hline Onion/Garlic & $30-75$ & Bulb Initiation \\
\hline Cabbage/Cauliflower & $30-45$ & Head initiation \\
\hline Okra & $15-30$ & $10-15 \mathrm{~cm}$ tall \\
\hline Tomato/Chilli & $30-45$ & $20-33 \mathrm{~cm}$ tall \\
\hline Brinjal & $20-60$ & ---- \\
\hline Beans & ---- & Canopy Formation \\
\hline Potato/Radish & $25-30$ & ---- \\
\hline Carrot & $15-20$ & $7-10 \mathrm{~cm}$ tall \\
\hline
\end{tabular}

Source: Singh et al., (1993)

Table.7 Effect of drip and furrow irrigations on weed intensity in cabbage

\begin{tabular}{|l|c|c|c|c|}
\hline \multirow{2}{*}{ Days after transplanting } & \multicolumn{2}{|c|}{${\text { Number of weeds } \mathbf{~ m}^{-2}}^{2}$} & \multicolumn{2}{c|}{ Dry wt. of weeds $\mathbf{( g m}^{-2}$ ) } \\
\cline { 2 - 5 } & Drip & Furrow & Drip & Furrow \\
\hline $\mathbf{3 0}$ & 1409 & 1844 & 128 & 157 \\
\hline $\mathbf{6 0}$ & 948 & 1530 & 129 & 170 \\
\hline $\mathbf{8 0}$ & 352 & 1165 & 44 & 61 \\
\hline
\end{tabular}

Source: Kudarimani (1977)

Table. 8 On the basis of chemical

1a. Inorganic

\begin{tabular}{|l|l|}
\hline \multicolumn{1}{|c|}{ Group } & \multicolumn{1}{c|}{ Chemical } \\
\hline Acids & Sulphuric acid, Arsenic acid \\
\hline Chlorate & Sodium chlorate, Borax, Decahydarate, Sodium metaborate \\
\hline Sulphamate & Copper sulphate, Ferric sulphate \\
\hline AMS & Ammonium sulphamate \\
\hline Nitrate & Sodium nitrate \\
\hline
\end{tabular}

1b. Organic herbicides

\begin{tabular}{|l|l|}
\hline Group & Herbicide Name \\
\hline Aliphatics & Dalapon,TCA \\
\hline Amides and Anillides & Alachlor, Butachlor, Propachlor, Propanil, Naptalam, Acrolein \\
\hline Anilines and Nitro-phenols & Dintramine, Nitralin, Triflutrlin, Fluchoralin, Nitrofen \\
\hline Arsenicals & DSMA, MA, MSMA \\
\hline Benzoics and Phenyl Acetic Acid & Chloramben, Dicamba, Fenac \\
\hline Carbamate & Diclormate, Asulam, Barban, Propham \\
\hline Thio-Carbamate & Benthiocarb, EPTC, Diallate, Tra-allate, Molinate, Glyphosate \\
\hline Heterocyclic Compounds & $\begin{array}{l}\text { Bipyridyelium, Pyridines, Pyridazines, Uracils, Atrazine, Simazine, Propazine, } \\
\text { Ametryne, Promatone, Terbutryn, Metribuzin }\end{array}$ \\
\hline Misc. Heterocyclic Compounds & Aminotriazole \\
\hline Hormone & Phenoxy acetic acid, Phenoxy propoinic acid, Phenoxy butyric acid \\
\hline Nitriles & Bromoxynil, Dichlobenil, Loxynil \\
\hline Substituted Urea & Chloroxuron, Diuron, Fenuron, Fluomrturon, Monuron \\
\hline Alkoxy & Liuron, Chlorbromuron, Neburon \\
\hline Unclassified & Methazole, Perfluidon, C-288 \\
\hline Nitriles & Bromoxynil, Dichlobenil, Loxynil \\
\hline Source: Brin (1964) & \\
\hline
\end{tabular}

Source: Brian (1964) 
Table.9 Commonly used herbicides in vegetables

\begin{tabular}{|l|l|l|c|l|}
\hline Common name & Trade name & $\begin{array}{l}\text { Time of } \\
\text { application }\end{array}$ & $\begin{array}{l}\text { Rate }_{\left(\mathbf{k g l}^{-1}\right.} \\
\left.\mathbf{h a}^{-1}\right)\end{array}$ & Usages \\
\hline Alachlor & Lasso & Pre- & $2-3$ & Selective \\
\hline Atrazine & Atrataf & Pre- & $0.5-2$ & Selective \\
\hline Borate & Hibour, Monobar & Soil pre/post & $2-3$ & Non-selective \\
\hline Butachlor & Machete & Pre- & $1-2$ & Selective \\
\hline Fluchloralin & Basalin & Pre- & $1-2$ & Selective \\
\hline Gluphosate & Round up & Post & $1-2$ & Non-selective \\
\hline Metribuzin & Lexone, Sencor & Pre- & $0.25-1$ & Selective \\
\hline Nitrofen & Tok-E-25 & Pre/post & $2-5$ & Selective \\
\hline Oxadiazon & Ronstar & Post & $0.75-4$ & Selective \\
\hline Paraquat & Gramaxone & Post & $0.5-1$ & Non-selective \\
\hline Picloram & Tordon,Amdon & Pre/post & $2-4$ & Selective \\
\hline Diquat & Reglone & Post & $1-2$ & Non-selective \\
\hline Simazine & Gesatop & Pre & & Selective \\
\hline
\end{tabular}

Table.10 Pesticide use in India and world

\begin{tabular}{|l|c|c|c|}
\hline \multirow{2}{*}{ Pesticide } & \multicolumn{3}{c|}{ \% share of herbicides out of total pesticide consumption } \\
\cline { 2 - 4 } & India & World & USA \\
\hline Herbicide & 20 & 52 & 60 \\
\hline Insecticide & 61 & 7 & 25 \\
\hline Fungicide & 17 & 32 & 8 \\
\hline Others & 2 & 4 & --- \\
\hline
\end{tabular}

*Based on pesticide industry data 2008

Table.11 Selective herbicides for weed control in vegetable crops

\begin{tabular}{|l|l|l|l|}
\hline \multicolumn{1}{|c|}{ Herbicides } & $\begin{array}{l}\text { Dose } \\
\left.\mathbf{( k g ~ h a}^{-1}\right)\end{array}$ & \multicolumn{1}{|c|}{ Treatment } & \multicolumn{1}{c|}{ Crops } \\
\hline Pendimathelin & $0.65-1.0$ & Pre-emergence & $\begin{array}{l}\text { Transplanted pepper, onion, garlic, } \\
\text { spinach brassica crops, umbelliferous } \\
\text { crops, legumes and potato. }\end{array}$ \\
\hline $\begin{array}{l}\text { Fluchloralin/ } \\
\text { Trifluralin }\end{array}$ & $1.0-1.5$ & $\begin{array}{l}\text { Pre plant- } \\
\text { incorporation }\end{array}$ & $\begin{array}{l}\text { Transplanted tomato, pepper, brinjal, } \\
\text { potato, okra, brassica crops, legumes, } \\
\text { garlic and umbelliferous crops. }\end{array}$ \\
\hline Oxyfluoren & $0.24-0.36$ & $\begin{array}{l}\text { Early post- } \\
\text { emergence }\end{array}$ & $\begin{array}{l}\text { Direct seeded and transplanted onion } \\
\text { and potato. }\end{array}$ \\
\hline Butachlor & 2.0 & Pre-emergence & Transplanted tomato \& cucurbits. \\
\hline Metribuzin & $0.2-0.35$ & $\begin{array}{l}\text { Pre or early post } \\
\text { emergence }\end{array}$ & $\begin{array}{l}\text { Direct seeded and transplanted } \\
\text { tomato and potato. }\end{array}$ \\
\hline
\end{tabular}

Source: Yaduraj and Dubey (2002) 
Table.12 Examples of promising bio-agents in weeds

\begin{tabular}{|l|l|l|l|}
\hline Weed & Bioagent & $\begin{array}{l}\text { Reporting } \\
\text { country }\end{array}$ & Kind of bioagent \\
\hline $\begin{array}{l}\text { Chondrilla } \\
\text { juncea }\end{array}$ & Puccinia chondrillina & Australia & Plant Pathogen \\
\hline Cirsium arvensis & Septoria cirsii & Plant Pathogen \\
\hline Cyperus rotundus & Bactra verutana & $\begin{array}{l}\text { India, } \\
\text { Pakistan } \\
\text { \& USA }\end{array}$ & $\begin{array}{l}\text { Shoot boring } \\
\text { moth }\end{array}$ \\
\hline $\begin{array}{l}\text { Eupatorium } \\
\text { riparium }\end{array}$ & Entyloma compositarum & USA & Plant pathogen \\
\hline $\begin{array}{l}\text { Hydrilla } \\
\text { verticillata }\end{array}$ & Hydrillaq pakistanae & USA & Shoot fly \\
\hline $\begin{array}{l}\text { Orobanche } \\
\text { cermua }\end{array}$ & Sclerotina spp. & USA & Plant pathogen \\
\hline $\begin{array}{l}\text { Parthenium } \\
\text { hysterophorus }\end{array}$ & $\begin{array}{l}\text { (i) Zygograma bicolorata } \\
\text { (ii) } \text { Epiblema sternuana }\end{array}$ & $\begin{array}{l}\text { India } \\
\text { Australia } \\
\text { Australia }\end{array}$ & $\begin{array}{l}\text { Leaf eating insect } \\
\text { Stem galling } \\
\text { insect } \\
\text { Stem galling } \\
\text { insect }\end{array}$ \\
\hline Rumex spp. & (i) Uromyces rumicis & $\begin{array}{l}\text { USA } \\
\text { (ii) Gastrophysa viridula }\end{array}$ & $\begin{array}{l}\text { Plant pathogen } \\
\text { Beetle }\end{array}$ \\
\hline Tribulus terrestris & (i) $\begin{array}{l}\text { Uicrolarinus larevnii } \\
\text { (ii) M. lypriformis }\end{array}$ & USA & Pod weevil \\
\hline
\end{tabular}

Source: Parsad and Kumar (1999)

Table.13 Some commercial mycoherbicides (bio-herbicides) in use

\begin{tabular}{|l|l|l|}
\hline Product & Content & Weed Control \\
\hline De-Vine & $\begin{array}{l}\text { A liquid suspension of fungal spores of } \\
\text { Phytophthora palmivora Butlar. It causes } \\
\text { root rot in weeds }\end{array}$ & $\begin{array}{l}\text { Stranglevine (Morrenia } \\
\text { odorata) in citrus orchards }\end{array}$ \\
\hline Collego & $\begin{array}{l}\text { A suspension of fungal spores of } \\
\text { Colletotrichum gloeosporioides sub sp. } \\
\text { aeschynomone. }\end{array}$ & $\begin{array}{l}\text { Jointvetch (Aeschynomone } \\
\text { sp.) in rice field. It cause } \\
\text { stem and leaf blight in } \\
\text { weeds. }\end{array}$ \\
\hline Bipolaris & $\begin{array}{l}\text { A suspension of fungal spores of Bipolaris } \\
\text { sorghicola. }\end{array}$ & $\begin{array}{l}\text { Johnsongrass } \\
\text { (Sorghum helepense). }\end{array}$ \\
\hline Biolophos & $\begin{array}{l}\text { A microbial toxin produced as } \\
\text { fermentation product of Streptomyces } \\
\text { hygroscopicus }\end{array}$ & $\begin{array}{l}\text { Non-specific and general } \\
\text { vegetation. }\end{array}$ \\
\hline
\end{tabular}

Source: Parsad and Kumar (1999) 
Table.14 Use of herbicides in vegetables

Potato

\begin{tabular}{|l|l|l|l|}
\hline $\begin{array}{l}\text { Herbicide } \\
\text { (Trade name) }\end{array}$ & $\begin{array}{l}\text { Dose } \\
\text { (g ai ha }^{-\mathbf{1}} \text { ) }\end{array}$ & $\begin{array}{l}\text { Application } \\
\text { time }\end{array}$ & Remarks \\
\hline $\begin{array}{l}\text { Butachlor (Machete, } \\
\text { Dhanuchlor, Teer, } \\
\text { Donmix) }\end{array}$ & $750-1000$ & $\begin{array}{l}\text { Pre- } \\
\text { emergence }\end{array}$ & $\begin{array}{l}\text { Apply within 3-4 days of planting potato } \\
\text { tubers. Ensure adequate soil moisture at } \\
\text { the time of application, either before or } \\
\text { after irrigation. Controls many annual } \\
\text { grasses and some BLWs. }\end{array}$ \\
\hline $\begin{array}{l}\text { Fluchloralin } \\
\text { (Basalin) }\end{array}$ & $1000-1500$ & Pre-planting & $\begin{array}{l}\text { Controls many annual grasses and some } \\
\text { BLWs. }\end{array}$ \\
\hline Metribuzin (Sencor) & 525 & $\begin{array}{l}\text { Pre-emergence } \\
\text { or early post- } \\
\text { emergence }\end{array}$ & $\begin{array}{l}\text { Apply 3-4 days after planting or as post- } \\
\text { emergence when potato has reached 5 cm } \\
\text { in height. Control many annual weeds } \\
\text { both grasses and BLWs. }\end{array}$ \\
\hline $\begin{array}{l}\text { Pendimethalin } \\
\text { (Stomp, Pendistar) }\end{array}$ & 1000 & $\begin{array}{l}\text { Pre- } \\
\text { emergence }\end{array}$ & $\begin{array}{l}\text { Apply at 2-3 days after planting. Dose not } \\
\text { control emerged weeds. Controls many } \\
\text { annual grasses and some BLWs. }\end{array}$ \\
\hline
\end{tabular}

Cabbage/cauliflower

\begin{tabular}{|l|l|l|l|}
\hline $\begin{array}{l}\text { Herbicide } \\
\text { (Trade name) }\end{array}$ & $\begin{array}{l}\text { Dose } \\
\text { (g ai ha }^{-\mathbf{1}} \text { ) }\end{array}$ & $\begin{array}{l}\text { Application } \\
\text { time }\end{array}$ & Remarks \\
\hline $\begin{array}{l}\text { Fluchloralin } \\
\text { (Basalin) }\end{array}$ & 1000 & $\begin{array}{l}\text { Pre or post } \\
\text { transplanting }\end{array}$ & $\begin{array}{l}\text { Could be applied just before planting } \\
\text { followed by irrigation. Controls many } \\
\text { annual grasses and some BLWs. }\end{array}$ \\
\hline $\begin{array}{l}\text { Pendimethalin } \\
\text { (Stomp,Pendistar) }\end{array}$ & 1000 & $\begin{array}{l}\text { Pre or post } \\
\text { transplanting }\end{array}$ & $\begin{array}{l}\text { Apply before or immediately after } \\
\text { transplanting followed by irrigation. } \\
\text { Controls many annual grasses and } \\
\text { some BLWs. }\end{array}$ \\
\hline
\end{tabular}

Radish/Carrot

\begin{tabular}{|l|l|l|l|}
\hline $\begin{array}{l}\text { Herbicide } \\
\text { (Trade name) }\end{array}$ & $\begin{array}{l}\text { Dose } \\
\text { (g ai ha }^{-\mathbf{1}} \text { ) }\end{array}$ & $\begin{array}{l}\text { Application } \\
\text { time }\end{array}$ & Remarks \\
\hline $\begin{array}{l}\text { Fluchloralin } \\
\text { (Basalin) }\end{array}$ & 1000 & $\begin{array}{l}\text { Pre -planting } \\
\text { or post } \\
\text { transplanting }\end{array}$ & $\begin{array}{l}\text { Apply before planting in case of } \\
\text { direct seeded crop. Apply before or } \\
\text { after transplanting followed by } \\
\text { irrigation. Controls many annual } \\
\text { grasses and some BLWs. }\end{array}$ \\
\hline $\begin{array}{l}\text { Pendimethalin } \\
\text { (Stomp, } \\
\text { Pendistar) }\end{array}$ & 1000 & $\begin{array}{l}\text { 3-5 DAS or } \\
\text { Pre or post } \\
\text { transplanting }\end{array}$ & $\begin{array}{l}\text { Apply from 1-3 DAS in case of } \\
\text { direct seeded crop. Application } \\
\text { before or after transplanting followed } \\
\text { by irrigation. Controls many annual } \\
\text { grasses and some BLWs. }\end{array}$ \\
\hline
\end{tabular}


Tomato/Brinjal/Chilli/Green Pepper

\begin{tabular}{|c|c|c|c|}
\hline $\begin{array}{l}\text { Herbicide } \\
\text { (Trade name) }\end{array}$ & $\begin{array}{l}\text { Dose } \\
(\mathrm{g} \text { ai ha } \\
\end{array}$ & $\begin{array}{l}\text { Application } \\
\text { time }\end{array}$ & Remarks \\
\hline $\begin{array}{l}\text { Alachlor } \\
\text { (Lasso) }\end{array}$ & 2000 & $\begin{array}{l}\text { Pre - } \\
\text { transplanting }\end{array}$ & $\begin{array}{l}\text { Apply before transplanting followed } \\
\text { by irrigation. Controls many annual } \\
\text { grasses and some BLWs. }\end{array}$ \\
\hline $\begin{array}{l}\text { Fluchloralin } \\
\text { (Basalin) }\end{array}$ & 1000 & $\begin{array}{l}\text { Pre - } \\
\text { transplanting }\end{array}$ & $\begin{array}{l}\text { Apply before transplanting followed } \\
\text { by irrigation. Controls many annual } \\
\text { grasses and some BLWs. }\end{array}$ \\
\hline $\begin{array}{l}\text { Pendimethalin } \\
\text { (Stomp, } \\
\text { Pendistar) }\end{array}$ & 1000 & $\begin{array}{l}\text { Pre or post } \\
\text { transplanting }\end{array}$ & $\begin{array}{l}\text { Controls many annual } \\
\text { grasses and some BLWs. }\end{array}$ \\
\hline $\begin{array}{l}\text { Quizalofop-p-ethyl } \\
\text { (Targa super) }\end{array}$ & 50 & $\begin{array}{l}\text { Post - } \\
\text { emergence }\end{array}$ & Kills many grassy weeds. \\
\hline
\end{tabular}

\section{Onion/Garlic}

\begin{tabular}{|l|l|l|l|}
\hline $\begin{array}{l}\text { Herbicide } \\
\text { (Trade name) }\end{array}$ & $\begin{array}{l}\text { Dose } \\
\text { (g ai ha }^{-\mathbf{1}} \text { ) }\end{array}$ & $\begin{array}{l}\text { Application } \\
\text { time }\end{array}$ & Remarks \\
\hline $\begin{array}{l}\text { Butaclor } \\
\text { (Machete, Bilchlor) }\end{array}$ & $1000-1250$ & $\begin{array}{l}\text { Post - } \\
\text { transplanting }\end{array}$ & $\begin{array}{l}\text { Apply at 3-4 days before or after } \\
\text { transplanting planting. Does not } \\
\text { control emerged weeds. Controls } \\
\text { many annual grasses and some } \\
\text { BLWs. }\end{array}$ \\
\hline $\begin{array}{l}\text { Fluchloralin } \\
\text { (Basalin) }\end{array}$ & 1000 & $\begin{array}{l}\text { Pre or post } \\
\text { transplanting }\end{array}$ & $\begin{array}{l}\text { Apply before or after transplanting } \\
\text { and followed by irrigation. Controls } \\
\text { many annual grasses and some } \\
\text { BLWs. }\end{array}$ \\
\hline Oxadiazon(Ronstar) & $500-700$ & $\begin{array}{l}\text { Pre or post } \\
\text { transplanting }\end{array}$ & $\begin{array}{l}\text { Apply before or after transplanting } \\
\text { followed by irrigation. Controls } \\
\text { many annual grasses and some } \\
\text { BLWs. }\end{array}$ \\
\hline $\begin{array}{l}\text { Pendimethalin } \\
\text { (Stomp,Pendistar) }\end{array}$ & 1000 & $\begin{array}{l}\text { Pre or post } \\
\text { transplanting }\end{array}$ & $\begin{array}{l}\text { Apply before transplanting followed } \\
\text { by irrigation. Controls many annual } \\
\text { grasses and some BLWs. If directly } \\
\text { sown, spray within 2-3 days of } \\
\text { sowing. Apply 0-3 DAS in direct- } \\
\text { seeded crop. }\end{array}$ \\
\hline
\end{tabular}

Source: Herbicide Use in Field Crops (2009)

\section{Chemical weed control}

The first attempt was made to control the weeds by herbicide in Punjab during 1937,

For controlling Carthamus oxycantha by using Sodium arsenate.

\section{Advantages}

Herbicides are not beneficial but profitable where labour is scarce or expensive.

Control weeds in crop rows where cultivation is not possible. 
Pre-emergence herbicides provide early season weed control when competition results in the greatest yield reduction and when other methods are less efficient.

Herbicide reduces the destruction of soil structure by decreasing the need for tillage.

They reduce fertilizers and irrigation requirements by eliminating competing weeds.

\section{Disadvantages}

Some herbicide persist in the environment Undeniable mammalian toxicity Selective herbicides control some weed only Are often inconsistent in weed control Have phytotoxicity effect

\section{Methods of Herbicide application}

\section{Pre-planting/pre-sowing}

The herbicides are applied in the seed-bed or in the field, incorporating in the soil,

Usually 20-30 days prior to planting or transplanting in the main field, so as to kill most of the weed seeds.

\section{Post-planting}

The herbicides are sprayed after planting the crop.

\section{Pre-emergence}

The treatment is made prior to the emergence of specific weeds. Mostly contact herbicides are used in this method.

The weedicides are applied after the weeds have emerged before the crop emergence and used an efficient herbicide that does not persist in toxic form in the soil.

\section{Post-emergence}

The treatment is given after the emergence of specific crop or weed; especially postemergence of the crop.

\section{Classification of herbicides}

\section{On the basis of selectivity}

\section{Selective}

Those herbicides which affect only certain weeds, leaving certain crops unharmed.

But the selectivity depends on the amount of chemical applied and the way they are used.

\section{Non - selective}

Are used to control a wide range of vegetation indiscriminately because they are toxic to all plants and highly susceptible to living plant tissues

\section{Biological weed control}

It is defined as the action of the parasite, predators or pathogens in maintaining other organism s population at a lower average density than would occur in their absence. The term was used by Smith (Debach, 1964).

\section{Advantages}

Reasonably permanent.

No harmful side effect.

No additional inputs are required once agent is established successfully.

Control often dependent on host density

Costs are no renewing

Risk are known and evaluated before use. 
Self dispersing spread to suitable host habitats.

High benefit: cost ratio for successful programs.

Self-perpetuating type.

\section{Disadvantages}

Initial cost of time, money and personnel is very high.

Does not work well in short term cropping cycles.

No guarantee of results.

Weed must be highly specialized

Control is low.

Establishment may fail for many reasons.

There may unknown ecological effects. Mutation to an undesirable form is possible.

If target is related to a crop, the number of potential biocontrol agent is low.

\section{Biocontrol agents}

\section{Classical or inoculative}

It has been used for many years.

The earliest record of biological weed control was the release of cochineal insect Dactylopius ceylonicus from Brazil to north India in 1795 to control prickly pear cactus.

\section{Inundative or augmentative}

E.g. Fungi (Colletotrichum gloeosporioides)

\section{Broad spectrum}

Fish (e.g. White amur or Grass carp (Ctenopharyngodon idella Valenciennes)

Aquatic Mammals e.g. Sea manatee (Trichechus spp.)

Vertebrates e.g. Sheep, goats etc.

Integrated weed management strategy for specific vegetable crops

Some advanced agricultural areas have developed integrated weed management systems. Some general strategies are summarized here (William et al., 2000).

\section{Green beans and peas}

Harvested legumes must be free of Solanum berries, thistle buds, Amaranthus stems, or crucifer pods. Crop rotations, close row spacings, early season weed control and cultivation (except in rocky or clod soils) are combined with herbicides to minimize weed competition and contamination of product.

A single post-emergence treatment can suppress weed competition or potential contamination of harvested peas.

\section{Carrots and celery}

Carrots suppress weeds when row spacings, population densities, cultivation and application of a single herbicide are combined. Cultivation also prevents sunburnt or green carrots roots by throwing soil over the roots.

\section{Table or red beets}

A combination of early season weed control, closely spaced rows, dense population, and cultivation will suppress mid- to late-season 
weed emergence after the crop canopy develops.

\section{Crucifer and cole crops}

Weed suppression in crucifers begins by rotating crops that demand different weed control practices to disrupt weed life cycles. Row spacing and plant density vary both to achieve head size, depending on the market, and in order to suppress weeds. Early-season weed control includes applying a herbicide and/or cultivation(s).

\section{Cucurbit crops}

Weed management in cucurbits means planning and integrating several practices. Crop rotations and pre-planting control of susceptible weeds must be carried out. Many growers practise stale seed beds followed by cultivation, except in excessively wet seasons. Row spacings that enhance canopy development and cultivation may be supplemented with a herbicide application within the crop row. Often rye windbreaks are planted between rows and incorporated during the last cultivation.

\section{Leaf crops}

Direct-seeded lettuce requires a couple of cultivations and a hand-thinning or weeding, whereas transplanted lettuce matures in 45 days following one or two cultivations with minor hand-weeding.

\section{Garlic and onion}

Garlic requires an almost perfect weed control since it emerges slowly, matures over a period of 10-11 months, and never forms a canopy with its short, vertical leaf arrangement. Growers, therefore, often control all weedy vegetation immediately prior to crop emergence, apply a selective soil-applied herbicide for winter weed control, and additional treatments are carried out during spring, depending on specific weed infestations. In onion, weeds are managed with selective herbicides combined with frequent cultivation. Winter cover crops enhance both soil and weed management.

\section{Tomato and pepper}

Weeds can be managed through preparatory tillage and a pre-planting herbicide in transplanted crops. Black plastic mulch can help to reduce the chemical need. Inter row tillage or post-emergence herbicide can control weeds later on. In direct-seeded crops, more intensive chemical treatments will be necessary. Management of Solanum nigrum (one of the worst weeds in tomato) should bear in mind the following points:

Chemical control in the previous crops where it is easier; (beet, carrot, celery, spinach);

That it prevails more in transplanted than in direct-seeded tomatoes;

Stale seed bed before tomato planting is recommendable;

Row application of soil-acting herbicides at planting (pendimethalin, oxifluorfen) integrated by inter row hoeing and/or by split low-dose treatments with metribuzin + rimsulfuron against $S$. nigrum at very early stages (up to two leaves) (Tei et al., 1999).

Weed control work has to be intensified in important vegetable crops to obtain maximum yield. Greater attention must be paid to integrated weed control practices instead opting a single practice. A careful watch has to be kept in places where one particular herbicide is being used continuously for a long time, because it may lead to growth of resistant weed species. Herbicide mixtures 
must be kept handy to control these resistant species. Extension services should be provided to farmers, so that the production system becomes more profitable.

\section{References}

Aldrich R.J. 1984. Weed crop ecology: principles in weed management. Berton Publishers. Massachusetts. 375p.

Brian R.C. 1964. The metabolism of herbicides. Weed Research 4(2): 105107.

Buchholitz K.P. 1967. Report of the terminology committee of the use science society of America. Weeds 15: 388-389.

Cooper J.I. and Harrison B.D. 1973. The role of weed host and the distribution and the activity of nematodes in the ecology of tobacco rattle virus. Annals of Applied Biology 73: 53-66.

DeBach P.1964. The scope of biological control. pp. 1-20. In "Biological control of insect pest and weeds". Reinhold, New York.

Forcella F., Benech R. L., Sanchez R. and Ghersa C.M. 2000. Modeling seedling emergence. Field Crops Res. 67: 23139.

Horowitz M., Regev Y. and Herzlinger G. 1983. Solarization for weed control. Weed Sci. 31: 170-179.

Kempen H.M. 1989. Weed management in vegetable crops. Growers Weed Management Guide. Thomson Publication. Fresno, Ca., USA, pp. 82158.

Kudarimani H.B. 1977. MSc (Ag.) Thesis. University of Agricultural Sciences, Bangalore.

Labrada R. 1996. Manejo de malezas en hortalizas. In: Labrada R, Caseley JC, Parker C. Manejo de malezas para países en desarrollo. Estudio FAO
Producción y Protección Vegetal 120. FAO, Roma. pp. 298-308.

Mallik R.K., Yadav A. and Rana M.K. 1998. Farmers and parliament, December Issue. (also available at http://weeds.ippc.orst.edu/pnw/weeds).

Pleasant J.M. and Schlather K. J. 1999. Weed Technology 8: 304-310.

Prasad S. and Kumar U.1999. In: Principle of horticulture, Agro Botanica. pp. 429430.

Singh J. and Dhankar B.S.1989. Vegetable Science 12:136-144.

Singh K., Panda M.C. and Jhakaral K.K.1993.Preceding of International Symposium. Indian Society of Weed Science, Hisar 1:365-368.

Tei F., Baumann D.T, Dobrzanski A., Giovinazzo R., Kleifeld Y., Roche F., Rzozi S.B., Sanseovic T. and Zaragoza C. 1999. Weeds and Weed Management in Tomato. A review. $11^{\text {th }}$ European Weed Research Society Symposium, Basel, Switzerland. 132 pp.

Temperini O., Bàrberi P., Paolini R., Campiglia E., Marucci A. and Saccardo F. 1998. Solarizzazione del terreno in serra-tunnel: effetto sulle infestanti in coltivazione sequenziale di lattuga, ravanello, rucola e pomodoro. In Proc. XI SIRFI Biennial Congress, Bari, Italy. 12-13 November, 213-228 (Italian, with English abstract).

Varshney J.G. 2009. Why weed control. Crop Care 35(10): 13-25.

William R.D., Ball D., Miller T.L., Parker R., Yenish J.P., Miller T.W., Morishita D.W. and Hutchinson P. 2000. Weed management in vegetable crops. Pacific Northwest Weed Control Handbook. Extension Services of Oregon St. University, Washington State University and University of Idaho. USA.pp.244-274.

Yaduraj N. I. and Dubey R. P. 2002. Compendium of lectures in winter 
school of recent advances in vegetable Production Technology held at IIVR, Varanasi, from 3-23 December, 2002. pp. 121-126.

Zaragoza C., Branthome X., Portugal J.M., Pardo A., Suso M., Rodríguez A., Monserrat A., Tiebas A., Fernández S. and Gutierrez M. 1994. Itineraires techniques compares pour le controle des mauvaises herbes chez la tomate en differentes regions europeennes. $5^{\text {th }}$ EWRS Mediterranean Symposium. Perugia, Italy. pp. 179-186.

\section{How to cite this article:}

Mandeep Singh, Amit Kaul, Vivek Pandey and Avtar Singh Bimbraw. 2019. Weed Management in Vegetable Crops to Reduce the Yield Losses.

Int.J.Curr.Microbiol.App.Sci. 8(07): 1241-1258. doi: https://doi.org/10.20546/ijcmas.2019.807.148 